\title{
Pregnancy-related changes in connections from the cervix to forebrain and hypothalamus in mice
}

\author{
Steven M Yellon, Lauren A Grisham, Genevieve M Rambau, Thomas J Lechuga \\ and Michael A Kirby \\ Departments of Physiology, Pediatrics, Obstetrics and Gynecology, Pathology and Human Anatomy, and the Center \\ for Perinatal Biology, School of Medicine, Loma Linda University, Loma Linda, California 92354, USA \\ Correspondence should be addressed to S M Yellon; Email: syellon@llu.edu
}

\begin{abstract}
The transneuronal tracer pseudorabies virus was used to test the hypothesis that connections from the cervix to the forebrain and hypothalamus are maintained with pregnancy. The virus was injected into the cervix of nonpregnant or pregnant mice, and, after 5 days, virus-labeled cells and fibers were found in specific forebrain regions and, most prominently, in portions of the hypothalamic paraventricular nucleus. With pregnancy, fewer neurons and fibers were evident in most brain regions compared to that in nonpregnant mice. In particular, little or no virus was found in the medial and ventral parvocellular subdivisions, anteroventral periventricular nucleus, or motor cortex in pregnant mice. By contrast, labeling of virus was sustained in the dorsal hypothalamus and suprachiasmatic nucleus in all groups. Based upon image analysis of digitized photomicrographs, the area with label in the rostral and medial parvocellular paraventricular nucleus and magnocellular subdivisions was significantly reduced in mice whose cervix was injected with virus during pregnancy than in nonpregnant mice. The findings indicate that connections from the cervix to brain regions that are involved in sensory input and integrative autonomic functions are reduced during pregnancy. The findings raise the possibility that remaining pathways from the cervix to the forebrain and hypothalamus may be important for control of pituitary neuroendocrine secretion, as well as for effector functions in the cervix as pregnancy nears term.

Reproduction (2010) 140 155-164
\end{abstract}

\section{Introduction}

Innervation of the cervix is important for a variety of physiological process related to reproduction and nociception. Afferent fibers in the cervix convey sensory information to the thoracolumbar spinal cord through the hypogastric, pelvic, and vagus nerves (Ortega-Villalobos et al. 1990, Steinman et al. 1992, Cueva-Rolon et al. 1996, Komisaruk et al. 1996, Papka et al. 1998, Lee \& Erskine 2000, Puder \& Papka 2001, Wiesel et al. 2004). These nerves mediate important functions related to pain perception, neuroendocrine secretion of oxytocin, and the fetal expulsion. By contrast to the marked decrease in innervation of the uterus with pregnancy (Wikland et al. 1984, Haase et al. 1997, Klukovits et al. 2002), the cervix is well innervated in individuals that are not pregnant, as well as throughout pregnancy and near term in a variety of species (Bryman et al. 1987, Tingaker et al. 2006). Moreover, in rodents, the greatest presence of nerve fibers in the cervix is found by the day before birth compared to that earlier in pregnancy or in nonpregnant controls (Kirby et al. 2005, Yellon et al. 2008, Boyd et al. 2009). The increase in nerve fibers in the preterm cervix temporally correlates with processes that are hypothesized to be critical for remodeling of the cervix, including immigration of immune cells, degradation of extracellular collagen matrix, and changes in biophysical capabilities to allow sufficient stretch for birth (Leppert 1995, Mackler et al. 1999, Yellon et al. 2003, Buhimschi et al. 2004). Innervation is also important for the birth process because remodeling of the cervix is forestalled and parturition disrupted after transection of the parasympathetic pelvic nerve (Higuchi et al. 1987, Mackay et al. 2009) or vagus nerve (Yellon et al. 2009), but not the sympathetic hypogastric nerve (Boyd et al. 2009). The potential importance of innervation for the birth process is countered by a lack of information from neural tract tracing studies of connections with the cervix during pregnancy.

$\mathrm{CN}$ innervation of the uterine cervix has been extensively studied in nonpregnant females. Areas of the forebrain and hypothalamus receive input from the lower thoracic and upper lumbar spinal cord either directly or via relays in the brainstem (Burstein et al. 1990, Lee \& Erskine 2000). These nuclei in the brainstem also project to the pelvic viscera through the vagus nerve (Ortega-Villalobos et al. 1990, Hubscher \& Berkley 1995). Recent evidence 
indicates that sensory and autonomic connections from the thoracolumbar spinal cord to the murine cervix are reduced with pregnancy (Kirby et al. 2009). Fewer cells and fibers in the spinal cord that project to the cervix during pregnancy suggest that processing and transmission of information to the brain are reduced. However, connections between the cervix and the intermediolateral column, a descending pathway in the autonomic region of the spinal cord, were sustained with pregnancy. Accordingly, the main objective of the present study was to determine the extent to which $\mathrm{CN}$ connections are maintained with the cervix during pregnancy. The retrograde transport of pseudorabies virus was used to identify brain areas that are directly or indirectly connected to primary neurons with terminal fibers in the cervix. This virus has served as a transneuronal tracer because it is rapidly taken up by nerve terminals, transported to the cell nucleus for replication, and, in the process, infect other neurons that synapse on virus-containing neurons (Card et al. 1993, Toth \& Palkovits 1997, Enquist 2002).
This approach was used to test the hypothesis that neural connections between the brain and cervix are reduced with pregnancy. The findings suggest a general reduction in central pathways that innervate the cervix with pregnancy and raise the possibility that remaining connections from specific locations may be important in the process of parturition.

\section{Results}

Cell bodies and neuronal processes in the brain were labeled following injection of pseudorabies virus into the cervix. Labeled neurons had a variety of morphologies, while fine fibers represented dendritic projections or thin axonal projections (Fig. 1). These structures were well preserved and counterstained, helping to identify relevant neuroanatomical landmarks. Immunoreactive particles partially or completely filled neuron cell bodies and their processes (Fig. 1 insets); isolated fibers with beaded varicosities were often evident. Clustering or
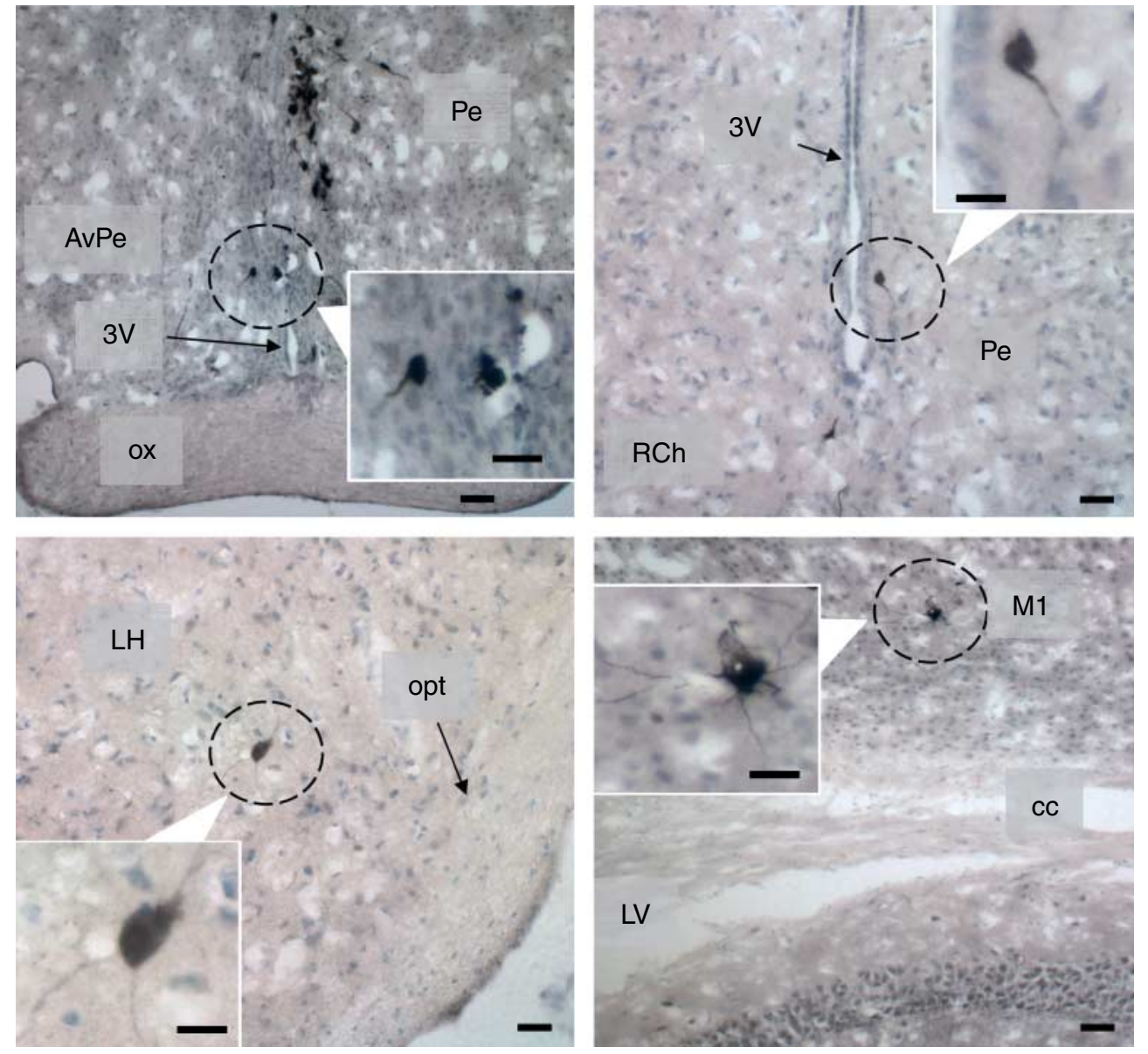

Figure 1 Photomicrographs of cells and fibers in forebrain and hypothalamus after the cervix of mouse \# 8 was injected with pseudorabies virus on day 15 of pregnancy. Top panels are anteroventral periventricular area (AvPe) and periventricular region (Pe), medial preoptic area (MPA), and retrochiasmatic area $(\mathrm{RCh})$. Bottom panels are lateral hypothalamus $(\mathrm{LH})$ and sensorimotor cortex $(\mathrm{M} 1)$. Other neuroanatomical structures are optic chiasm (ox), third ventricle (3V), corpus callosum (cc), and lateral ventricle (LV). Insets illustrate fine fibers that emanate from soma and diversity of somas that were labeled with virus. Scale bar is $50 \mu \mathrm{m}$ for all panels and $25 \mu \mathrm{m}$ for all insets. 

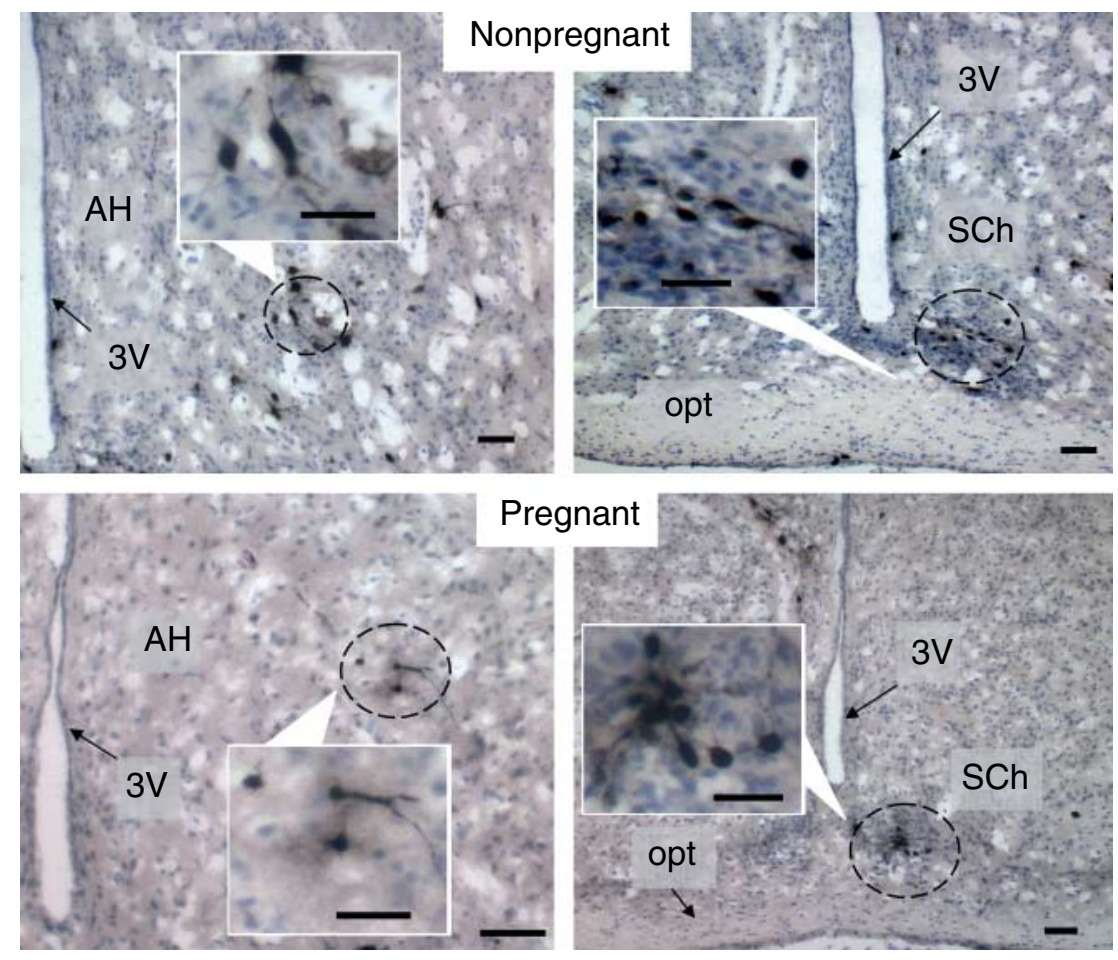

Figure 2 Photomicrographs of cells and fibers in forebrain and hypothalamus after the cervix of a nonpregnant mouse (top panels) or day 15 pregnant mouse (bottom panels) was injected with pseudorabies virus. Left panels are from the anterior hypothalamus (AH). Right panels are of the suprachiasmatic nuclei (SCh). Other neuroanatomical structures are the third ventricle (3V) and optic tract (opt). Insets highlight morphology of cells and fibers labeled with virus. Scale bar is $50 \mu \mathrm{m}$ for all panels and $25 \mu \mathrm{m}$ for all insets. overlap of morphological features precluded an accurate count of labeled neurons. Virus-labeled cell bodies and fibers were scattered or isolated in the periventricular area and various hypothalamic nuclei in all mice, as well as in the medial preoptic area in most mice. In each of these areas, immunoreactivity was more prevalent within and over multiple sections in nonpregnant controls compared to that in mice that had been injected with virus into the cervix during pregnancy. Viruslabeled cells and fibers were also present in the anteroventral periventricular area and sparse or isolated in the somatosensory and primary motor cortex in nonpregnant mice and in most mice in the day 15 groups, but absent in mice in the day 18 group. An example of diminished presence of label in the anterior hypothalamus with pregnancy is shown in Fig. 2 (left panels). By contrast, immunoreactive cell bodies and fibers were prevalent in the dorsomedial hypothalamus of both groups of pregnant mice, but less abundant in nonpregnant controls. Finally, in the suprachiasmatic nucleus of the hypothalamus, a few virus-labeled neuron cell bodies and/or fibers were consistently present in sections from all mice across groups, specifically in the dorsal apex and occasionally in medial portions of the nucleus (Fig. 2, right panels). Stain for pseudorabies virus was found sporadically in a few mice irrespective to group in the bed nucleus of the stria terminalis, retrochiasmatic area, and amygdala, but in no other locations.

The hypothalamic paraventricular nucleus was the most prominent area to contain pseudorabies virus.
Bilaterally, both parvocellular and magnocellular subdivisions had clusters of virus-labeled neuron cell bodies and fibers (Fig. 3). In nonpregnant mice, dense label was evident throughout the rostral-caudal expanse of the parvocellular paraventricular nucleus. The rostral paraventricular nucleus, corresponding to anterior and ventral parvocellular subdivisions, contained many immunoreactive cells and relatively large diameter fibers. Virus label was also well distributed in the middle portion of the paraventricular nucleus, encompassing the medial parvocellular, as well as lateral and medial magnocellular subdivisions. Caudally, labeled cells and fibers were prevalent in the posterior parvocellular subdivision of the paraventricular nucleus; however, virus was sparse or absent in the medial posterior parvocellular portion.

Compared to findings in nonpregnant mice, labeled neurons and processes were, in general, similarly distributed in the paraventricular nucleus of mice injected with virus into the cervix on day 15 or 18 of pregnancy. However, virus-labeled cells and fibers in most regions of the paraventricular nucleus were markedly reduced and more sparsely distributed in pregnant D15 and 18 groups. Pseudorabies virus was prominent, though markedly reduced in the rostral anterior parvocellular subdivision in the day 15 and 18 groups compared to that in nonpregnant mice. Few labeled cells were found in the ventral parvocellular portion in the day 15 group, and virus was nearly absent in this region in the day 18 group. In middle and caudal areas in groups whose cervix was injected with pseudorabies virus during pregnancy, the density of 

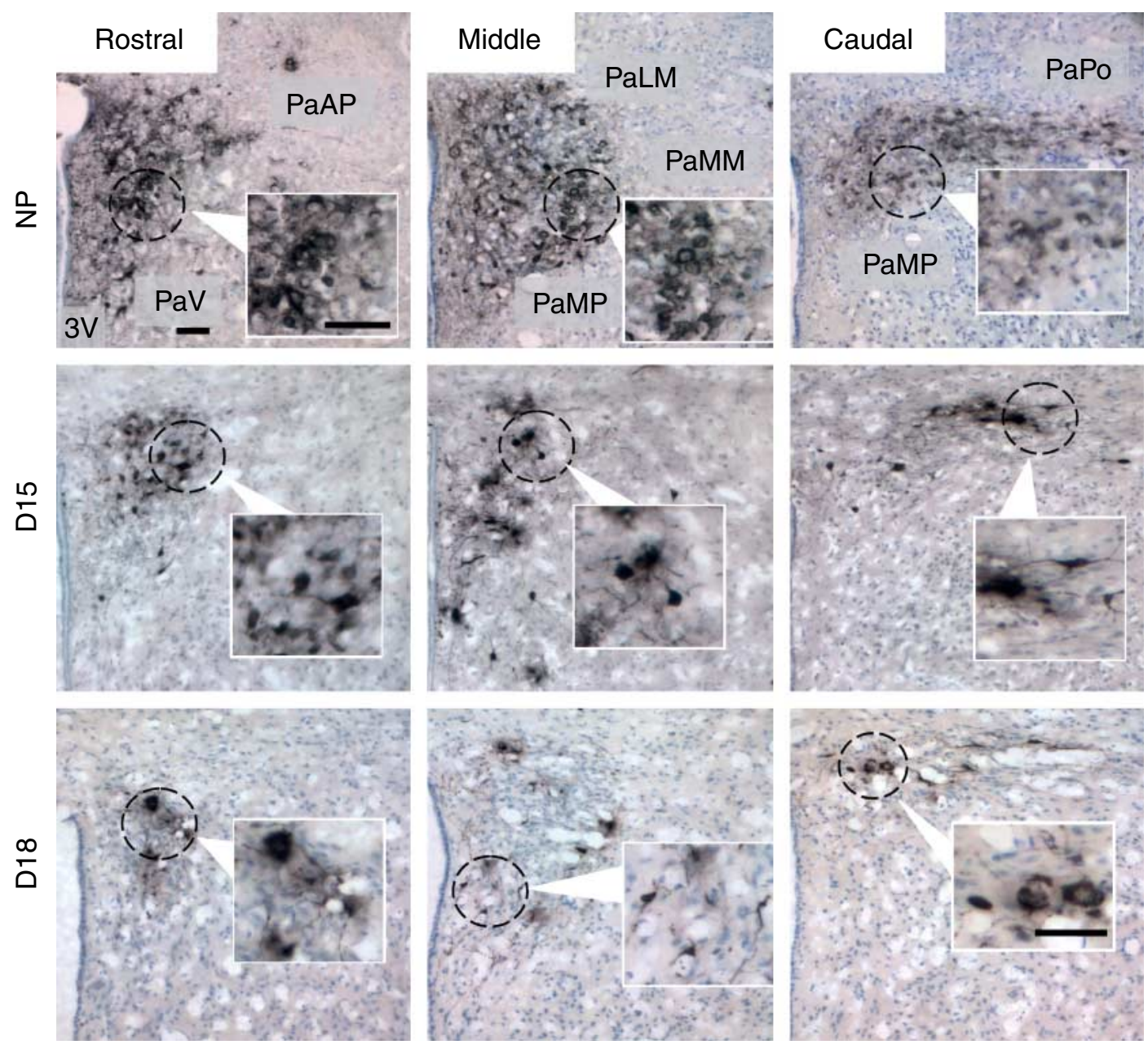

Figure 3 Photomicrographs of pseudorabies virus-labeled cells and fibers in the hypothalamic paraventricular nucleus 5 days after injection of virus into the cervix of nonpregnant mice, and mice on day 15 or 18 of pregnancy (top, middle, or bottom panels respectively). Based on the Paxinos \& Franklin (2004) mouse brain atlas, designations correspond to rostral (Figure 36), middle (Figures 38 and 39), and caudal (Figures 40 and 41 ) portions of the paraventricular nucleus. Insets highlight virus label in individual and clusters of cells, fine fibers, as well as single or multiple projections. Subdivisions of the paraventricular nucleus are parvocellular, i.e. ventral (PaV), anterior (PaAP), and posterior (PaPo), or magnocellular, i.e. lateral (PaLM), medial (PaMM), and posterior (PaMP). Scale bar is $50 \mu \mathrm{m}$ for all panels and $50 \mu \mathrm{m}$ for all insets.

labeled cells and fibers was reduced in lateral and medial magnocellular subdivisions, as well as in the posterior parvocellular subdivision relative to that in nonpregnant mice. In both middle and caudal regions, little or no virus was found in the medial parvocellular portion of the paraventricular nucleus in pregnant versus nonpregnant groups.

Pixelated images accurately reflected the area of pseudorabies virus-labeled neuron cell bodies and larger fibers in photomicrographs of the paraventricular nucleus among groups. Figure 4 represents digitized images for photomicrographs in Fig. 3. The area of virus-labeled structure was calculated from the number of pixels that met threshold criteria multiplied by the calibrated area/pixel. The sum total area of label in the paraventricular nucleus was reduced in mice that had pseudorabies virus injected into the cervix when pregnant compared to that in nonpregnant controls
$(P<0.05)$. In rostral, middle, and caudal regions of the paraventricular nucleus, the area that contained virus was not significantly different in day 15 vs 18 groups. Across the rostral and middle extent of the paraventricular nucleus, the area with virus was reduced in the day 15 and 18 groups compared to that in nonpregnant mice (Fig. 5). Although the area with virus in the caudal paraventricular nucleus similarly was lower, variability precluded statistical differences among groups $(P=0.42)$. Thus, major reductions in area of virus, a reflection of diminished infection and connections from the cervix, were mostly in rostral portions of the parvocellular subdivision and the magnocellular portions of middle parts of the paraventricular nucleus. The presence of virus in anterior and posterior parvocellular, as well as magnocellular subdivisions of the paraventricular nucleus indicates that specific connections with the cervix are sustained with pregnancy. 


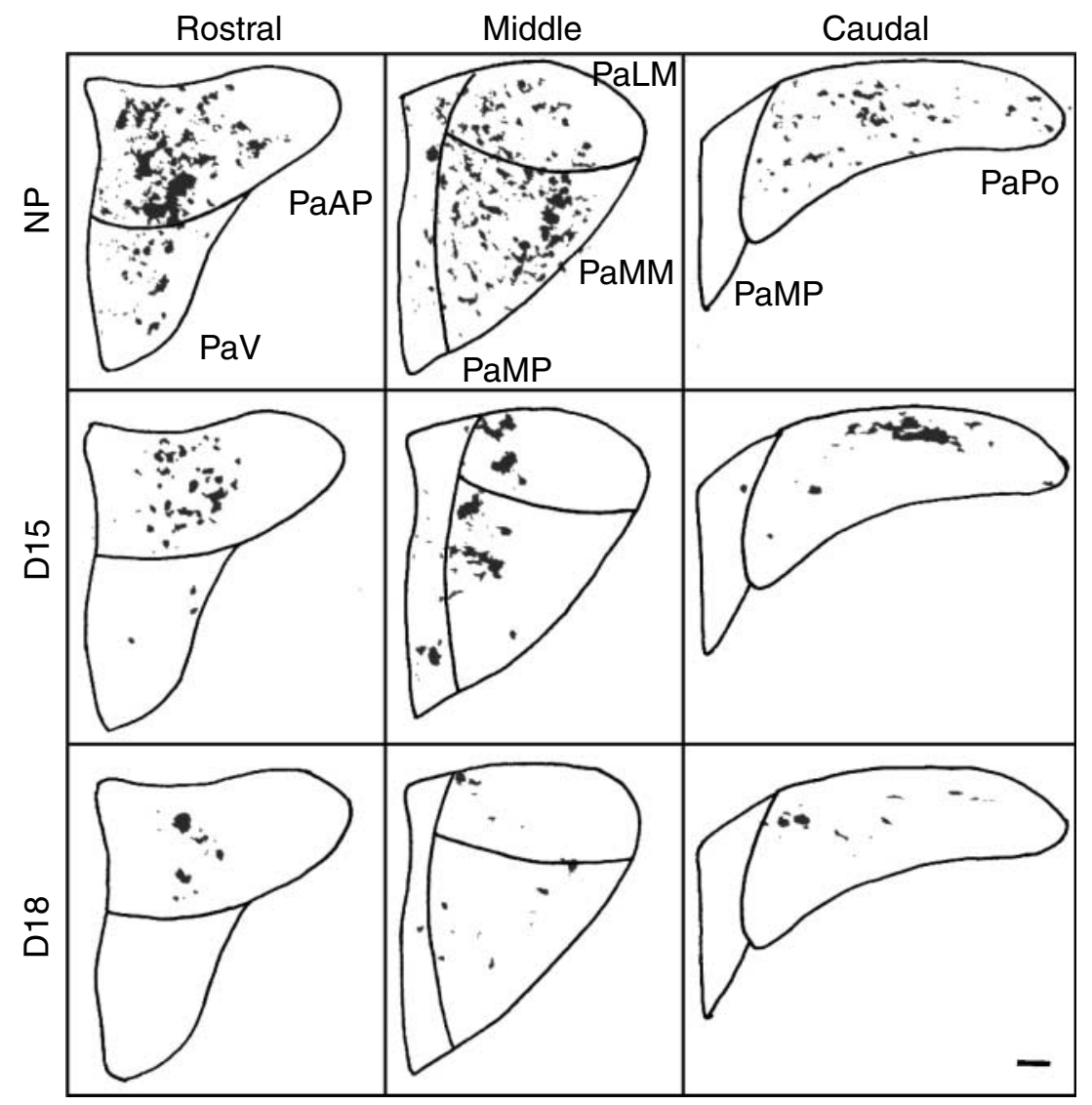

Figure 4 Digitized images of pseudorabies virus label in sections of paraventricular nucleus in Fig. 2 using Image Pro Plus 6 software. Subdivision designations were based upon the mouse atlas by Paxinos \& Franklin (2004). Pixels with pseudorabies virus label were counted to calculate the area of the paraventricular nucleus with cells and fibers that were infected 5 days after injection of virus into the cervix. Small structures and isolated thin fibers were often below the limit of detection. Scale bar is $50 \mu \mathrm{m}$.

\section{Discussion}

Pseudorabies virus has proven useful for the studies of multi-synaptic pathways from reproductive structures to the $\mathrm{CN}$. In the present study, a standardized method to deliver virus into the cervix revealed connections to specific brain locations, while image analyses provided replicable estimates of the area of pseudorabies virus

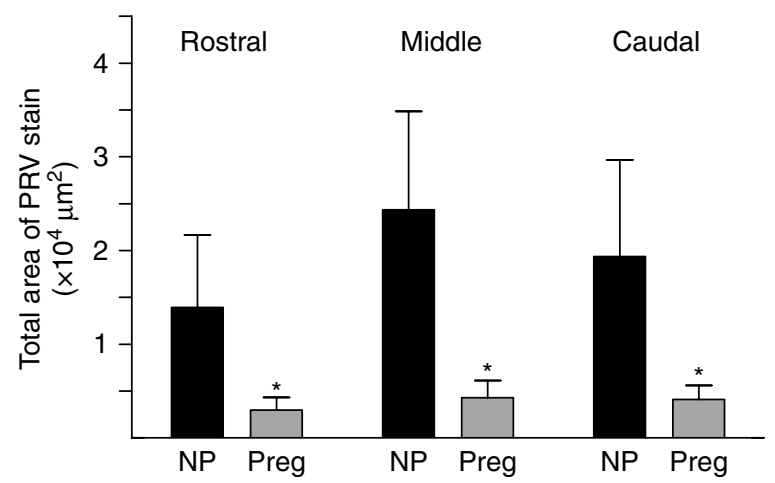

Figure 5 Area of PRV-labeled cells and fibers in subdivisions of paraventricular nucleus from nonpregnant mice (NP, $n=3)$ and mice injected with pseudorabies virus into the cervix on day 15 or 18 postbreeding (Preg, $n=6$ ). Data are the mean sum of pixels ( \pm S.E.M.) from Image Pro Plus 6-analyzed digitized images of photomicrographs (details in Materials and Methods). ${ }^{*}$ Indicates $P<0.05$ versus NP group. label within the paraventricular nucleus among individuals in different groups of mice. The presence of virus in rostral forebrain areas in nonpregnant and pregnant mice suggests that transport, rate of infection, and replication of virus were not affected by pregnancy. Evidence further suggests that supraspinal connections to the cervix in nonpregnant mice were comparable to previous studies in nonpregnant rats after pseudorabies virus was injected into both the uterus and cervix (Papka et al. 1998, Collins et al. 1999, Lee \& Erskine 2000). Thus, the present results are the first to indicate that the cervix, when injected with a retrograde tract tracer, is innervated by specific brain regions. With respect to pregnancy, injection of pseudorabies virus into a uterine horn resulted in the reduced number of labeled neurons in the brain compared to that in nonpregnant rats (Wiesel et al. 2004). This finding is consistent with evidence that the uterus is virtually denervated as pregnancy progresses. By contrast, the cervix remains well innervated throughout pregnancy (Kirby et al. 2005, Yellon et al. 2008, Boyd et al. 2009), a likely consequence of local proliferation of fibers in the cervix by fewer sensory and autonomic connections between the spinal cord and cervix as term approaches (Kirby et al. 2009). Reductions in virus-labeled cells and fibers in most brain regions with pregnancy in the present study 
are consistent with findings in the spinal cord. These results support the second major finding that fewer neurons and fibers in the forebrain and hypothalamus are part of the multi-synaptic pathway that innervates the cervix during pregnancy as compared to a more diverse and complex connectivity in nonpregnant mice.

The paraventricular nucleus was the most prominent part of the forebrain or hypothalamic pathway to innervate the cervix in mice. Reductions in the presence of virus in both day 15 and 18 groups suggest a significant decline in input from the cervix to this integrative brain center with pregnancy. Brightfield microscopy and semiquantitative image analyses indicated a reduced presence of label in the rostral parvocellular and magnocellular subdivisions of the paraventricular nucleus in mice injected with virus into the cervix compared to that in nonpregnant controls. Reduced label in the paraventricular nucleus as pregnancy nears term closely reflects the multi-fold reduction in connections between the cervix and thoracolumbar spinal cord (Kirby et al. 2009). Ascending pathways in the dorsal columns and lamina $X$ in the autonomic subdivision of the spinal cord are known to mediate pain perception in nonpregnant rats (Chadha \& Hubscher 2008) and women (reviewed by Komisaruk \& Sansone 2003). In addition, the small diameter of labeled fibers in the cervix (Boyd et al. 2009) as well as small size of somata and the location of labeled cell bodies in the substantia gelatinosa in the spinal cord (Rexed's Layer II) are suggestive of presumptive nociceptive fibers, such as $C$ class pain fibers (Giesler et al. 1984, Jancso 1992). The paraventricular nucleus and various forebrain nuclei receive projections from the medulla and thoracolumbar spinal cord (Schwanzel-Fukuda et al. 1984, Wagner \& Clemens 1991). In rodents, neurons in the parvocellular subdivisions of the paraventricular nucleus primarily express oxytocin or vasopressin (Silverman \& Pickard 1983, Armstrong 1995, Simerly 1995). Within the paraventricular nucleus, axons of parvocellular neurons extensively innervate other intrinsic parvocellular neurons and magnocellular portions of the nucleus (Van den Pol 1982). Various parvocellular subdivisions also project through the caudal portions of the paraventricular nucleus and dorsal hypothalamus into a long descending pathway to autonomic relay centers in the hindbrain of rodents (Sawchenko \& Swanson 1983, Silverman \& Pickard 1983). These relay centers, the nucleus of the solitary tract and dorsal vagal complex in particular, project to the intermediolateral column of the spinal cord and vagus nerve. Diminished or loss of connections from the cervix to the medial or ventral parvocellular paraventricular nucleus respectively with pregnancy suggests a major reduction in integration of sensory input and autonomic function as term approaches. In rats, such connections in the hindbrain integrate cardiovascular sensory input and regulate parasympathetic control of blood flow to peripheral organs
(Sawchenko \& Swanson 1983, Bittencourt et al. 1991, Papka et al. 1998). It remains to be determined whether neural input to the prepartum cervix has effector functions that regulate blood flow, or immigration of immune cells, or vascular permeability at parturition.

In magnocellular subdivisions of the paraventricular nucleus, neurons are topologically segregated and principally project to the median eminence and posterior pituitary. This projection constitutes a final common pathway through the periventricular region as part of the neuroendocrine reflex that controls oxytocin and vasopressin secretion (Swanson \& Sawchenko 1983). Reduced input to this nucleus may promote intranuclei synchronization of burst activity by neurons responsible for enhanced oxytocin secretion at term (Higuchi \& Okere 2002). Magnocellular cells also provide afferent fibers to the median eminence to affect anterior pituitary ACTH release (Zimmerman et al. 1977, Silverman \& Pickard 1983). However, a proportion of magnocellular neurons, most of which are immunoreactive for neuronal nitric oxide synthetase, project to the spinal cord to regulate sympathetic output to the periphery (Krukoff 1998, Nylen et al. 2001). This projection may contribute to the increased presence of nitric oxide synthetase-immunoreactive nerve fibers in the cervix by the day before birth (Kirby et al. 2005). Although the importance of a magnocellular nitrinergic pathway for remodeling of the preterm cervix has yet to be determined, substantial sensory and autonomic connections from the prepartum cervix to magnocellular neurosecretory cells are evident at the conclusion of pregnancy. Thus, fewer inputs may focus integration of signals in the magnocellular subdivision to affect posterior pituitary oxytocin release and, as part of the Ferguson reflex, promote uterine contractility for expulsion of the fetus (Akaishi et al. 1988, Russell et al. 2003).

A decline in sensory or autonomic input to the paraventricular nucleus could also account for diminished or loss of virus with pregnancy in the medial preoptic area, anteroventral periventricular area, somatosensory and primary motor cortex (M1), as well as in the anterior, lateral, and ventromedial hypothalamic nuclei. In rats, these areas are reported to project to the paraventricular nucleus and are involved in cardiovascular responses, fluid homeostasis, and electrolyte metabolism (Swanson \& Sawchenko 1983, Simerly 1995). A reduction in extrahypothalamic connections to the paraventricular nucleus raises the possibility that cervical remodeling and powerful contractions of labor may have a diminished effect on these physiological processes at term. In another respect, the loss of connections between the cervix and both medial preoptic area and anteroventral periventricular areas in pregnant groups may be of particular interest since these areas contain substantial numbers of cells that bind gonadal steroid hormones (Warembourg 1978, Numan et al. 1999, Francis et al. 2002). Lack of evidence 
for progesterone receptors in the paraventricular nucleus raises the possibility that reduced connections from regions that contain progesterone receptors and project to the paraventricular nucleus, along with a decline in progesterone in circulation at term (Virgo \& Bellward 1974), may contribute to a novel mechanism for central withdrawal of the actions of progesterone. The impact of a systemic progesterone withdrawal on neural regulation of processes associated with remodeling of the cervix and with parturition remains to be determined.

Importantly, a stable connection between the suprachiasmatic nucleus and the cervix appears to be maintained during pregnancy. Labeled cells and fibers were consistently found in the suprachiasmatic nucleus of the hypothalamus of all mice after the injection of virus into the cervix of nonpregnant and pregnant groups. The suprachiasmatic nucleus projects to portions of the parvocellular paraventricular nucleus as part of a descending oxytocinergic pathway to the intermediolateral column of the spinal cord (Swanson \& Cowan 1975, Klein et al. 1983, Papka et al. 1998, Buijs et al. 2003). The suprachiasmatic nucleus serves as the master pacemaker for neural and endocrine rhythms that mediate photoperiodic regulation of physiological functions that are season-specific (Weaver 1998). Both the onset of labor and hour of birth in humans, as well as many animal species, are related to time of day (Reppert et al. 1987, Longo \& Yellon 1988). Evidence in a variety of species suggests that a circadian timekeeping mechanism predisposes birth to occur during the inactive phase of the light-dark cycle. Sustained connections from the cervix to the hypothalamic suprachiasmatic nucleus during pregnancy suggest that circadian timekeeping information may, in addition to regulating relevant endocrine rhythms in pregnancy, e.g. the melatonin rhythm (Yellon \& Longo 1987, Apostolakis et al. 1993), directly affect timing of processes associated with remodeling of the cervix in preparation for birth.

In summary, evidence indicates a reduction in forebrain and hypothalamic innervation of the cervix with pregnancy. The decline in paraventricular nucleus connections with the cervix likely reflects reduced transmission and, conceivably, processing of sensory and autonomic information from the thoracolumbar spinal cord with pregnancy. Although reduced as pregnancy nears term, the remaining $\mathrm{CN}$ innervation of the cervix may be critical for various functions of the paraventricular nucleus which involve magnocellular control of pituitary neurosecretion and neuroendocrine activity, as well as parvocellular processing that regulates autonomic reflexes. The present findings also support the hypothesis that remaining $\mathrm{CN}$ connections with the cervix during pregnancy may contribute to circadian influences on neuroimmune processes that remodel the cervix and time parturition.

\section{Materials and Methods}

Adult $\mathrm{C} 3 \mathrm{H} / \mathrm{HeN}$ mice were purchased from Charles River Laboratories (Wilmington, DE, USA) and housed individually with free access to food and water. Procedures were the same as previously reported in a study of spinal cord connections with the cervix (Kirby et al. 2009). The experimental protocol was approved by the Loma Linda University Animal Care and Use Committee, as well as the Biosafety and Hazardous Materials according to NIH Guidelines for the care and use of laboratory animals.

\section{Procedures to trace connections between the cervix and brain}

Procedures in the present study follow those previously described (Kirby et al. 2009) and follow those used to retrogradely trace multi-synaptic pathways that innervate the uterine cervix (Papka et al. 1998, Lee \& Erskine 2000). Mice were anesthetized with Nembutal $(2 \mathrm{mg} / \mathrm{kg})$, secured on an operating platform, and fresh pseudorabies virus $(\sim 5 \mu \mathrm{l}$ of $1 \times 10^{6}$ pfu Bartha $K$ strain herpes $A$ virus, gift of original culture from Dr Lynn Enright) directly injected into the cervix using a specially designed vaginal speculum over a $15-\mathrm{min}$ period. After 5 days, mice were killed with $\mathrm{CO}_{2}$, and perfused through the heart with warm saline and then with $4 \%$ paraformaldehyde. A 5-day survival period was empirically determined to be sufficient to label hypothalamic neurons following injections of virus into the cervix and follows the same time course used by others to trace central innervation of peripheral structures (Strack \& Loewy 1990, Aston-Jones \& Card 2000). Brains were post-fixed overnight, immersed in $30 \%$ sucrose until saturated, and embedded in OCT (Sakura Finetek, Torrance, CA, USA), and every fourth cryostat section $(20 \mu \mathrm{m})$ was processed by immunohistochemistry to identify pseudorabies virus-labeled cells and fibers by immunohistochemistry. Protocol specifications were the same as those used previously for primary antibody (1:10 000 dilution; provided by Dr Lynn Enquist), biotinylated donkey anti-rabbit secondary antibody (1:600; Fitzgerald Industries International, Concord, MA, USA; formally Research Diagnostics Inc.), and a $0.32 \%$ biotin/avidin HRP (Vectastain Elite ABC Kit, Vector Labs, Burlingame, CA, USA). Sections not incubated with primary antisera served as a negative control. All sections were counterstained with cresyl violet acetate to identify cell nuclei and then coverslipped with Permount mounting medium.

Based upon cervix morphology, nonpregnant mice were in diestrus or early proestrus phase of the ovarian cycle, i.e. increased estradiol and relatively low progesterone in circulation compared to that during estrus or pregnancy. Immunoreactive pseudorabies virus was present in $85 \%$ of the brains of all mice that received cervical injections of virus. In the cervix, immunoreactive label was found exclusively in cells that morphologically resembled monocytic phagocytes; virus was absent from adherent tissue and immediate proximal sections of the uterine body. Injection of pseudorabies virus into the cervix of rodents does not interfere with overall health or processes associated with parturition 
including cervical ripening and the normal timing of birth; pregnant mice gave birth to viable pups by the morning of day 19 post-breeding, typical of a normal gestation and parturition.

\section{Image analysis of pseudorabies virus in forebrain and hypothalamus}

Brain sections were viewed under brightfield with a Zeiss Axio Imager A1 microscope. Photomicrographs were taken with an Apogee camera using a $10 \times$ objective. Neuroanatomical locations of pseudorabies virus-infected cells and their fibers, darkly stained structures against a relatively clear background of violet counterstained nuclei as described by Kobayashi (1998), were qualitatively assessed by comparison to murine brain atlases (Sidman et al. 1983, Paxinos \& Franklin 2004). To assess the area of cells and fibers that were virus-labeled, Image Pro Plus software (Version 6, Media Cybernetics, L.P. Silver Springs, MD, USA) was used to convert photomicrographs into a digitized image. Specific stain was differentiated from background based upon grayscale threshold settings for color, as well as minimum and maximum numbers of pixel clusters. This approach was originally based upon procedures of Cummings \& Cotman (1995) to quantify area of $\beta$-amyloid deposits in the brain of Alzheimer's patients, and avoids technical concerns related to variability in intensity of stain between sections or immunohistochemistry runs, as well as subjectivity in enumeration of neurons and fibers based upon morphological criteria.

In the present study, brain sections were analyzed in each mouse/group (two each for rostral, middle, and caudal regions). The number of pixels that were labeled times the calibrated area of $1.61 \mu \mathrm{m}^{3} /$ pixel in the $10 \times$ brightfield lens field of view was used to estimate the total area of pseudorabies virus in each region of interest. Settings for filter range and color selection (histogram) were consistently used for all sections to control for variability. Accuracy and replicability of data were determined to exceed $95 \%$ with respect to tests of comparisons at $20 \times$ and $40 \times$ power, as well as using the Image Pro Plus 6 eyedropper method to set color threshold in a series of sections that were compared between two independent investigators blind to the experimental design. Although pixelation of digitized images accurately reflected the density and distribution of virus label in photomicrographs of stained sections, the analysis is likely to underestimate the total area with virus because thin fibers and small punctate structures that were seen at higher magnification, e.g. partial cell bodies, dendrites, and small diameter axons, were typically below the limit of sensitivity for detection. For statistical analysis, image analysis data were compared by Student's $t$-test; $P<0.05$ was considered statistically significant.

\section{Declaration of interest}

The authors declare that there is no conflict of interest that could be perceived as prejudicing the impartiality of the research reported.

\section{Funding}

This study was supported by NIH grant HD054931, as well as by Richard Chinnock, MD, Chair, Department of Pediatrics, and $\mathrm{H}$ Roger Hadley, MD, the Dean of Loma Linda University School of Medicine.

\section{Acknowledgements}

We wish to thank Long Tran for help with processing tissue and data analyses, as well as the technical assistance of Roxanne Williams, David A Singh, Prashant Verma, and Dan Wong on the project. We appreciate the contribution of Dr James D Kettering, PhD, Professor of Microbiology, Loma Linda University School of Dentistry, and his research assistant, Raydolfo M Aprecio, for growing and titering fresh pseudorabies virus from an initial aliquot that was generously provided as a gift from Dr Lynn Enquist, PhD (Princeton University).

\section{References}

Akaishi T, Robbins A, Sakuma Y \& Sato Y 1988 Neural inputs from the uterus to the paraventricular magnocellular neurons in the rat. Neuroscience Letters 84 57-62.

Apostolakis EM, Rice KE, Longo LD, Seron-ferre M \& Yellon SM 1993 Time of day of birth and absence of endocrine and uterine contractile activity rhythms in sheep. American Journal of Physiology 264 E534-E540.

Armstrong WE 1995 Hypothalamic supraoptic and paraventricular nuclei. In The Rat Nervous System, pp 377-390. Ed. G Paxinos. San Diego, CA: Academic Press.

Aston-Jones G \& Card JP 2000 Use of pseudorabies virus to delineate multisynaptic circuits in brain: opportunities and limitations. Journal of Neuroscience Methods 103 51-61.

Bittencourt JC, Benoit R \& Sawchenko PE 1991 Distribution and origins of substance P-immunoreactive projections to the paraventricular and supraoptic nuclei: partial overlap with ascending catecholaminergic projections. Journal of Chemical Neuroanatomy 4 63-78.

Boyd JW, Lechuga TJ, Ebner CA, Kirby MA \& Yellon SM 2009 Cervix remodeling and parturition in the rat: lack of a role for hypogastric innervation. Reproduction 137 739-748.

Bryman I, Norstrom A, Dahlstrom A \& Lindblom B 1987 Immunohistochemical evidence for preserved innervation of the human cervix during pregnancy. Gynecologic and Obstetric Investigation 24 73-79.

Buhimschi IA, Dussably L, Buhimschi CS, Ahmed A \& Weiner CP 2004 Physical and biomechanical characteristics of rat cervical ripening are not consistent with increased collagenase activity. American Journal of Obstetrics and Gynecology 191 1695-1704.

Buijs RM, la Fleur SE, Wortel J, Van Heyningen C, Zuiddam L, Mettenleiter TC, Kalsbeek A, Nagai K \& Niijima A 2003 The suprachiasmatic nucleus balances sympathetic and parasympathetic output to peripheral organs through separate preautonomic neurons. Journal of Comparative Neurology 464 36-48.

Burstein R, Cliffer KD \& Giesler GJ Jr 1990 Cells of origin of the spinohypothalamic tract in the rat. Journal of Comparative Neurology 291 329-344.

Card JP, Rinaman L, Lynn RB, Lee BH, Meade RP, Miselis RR \& Enquist LW 1993 Pseudorabies virus infection of the rat central nervous system: ultrastructural characterization of viral replication, transport, and pathogenesis. Journal of Neuroscience 13 2515-2539.

Chadha HK \& Hubscher CH 2008 Convergence of nociceptive information in the forebrain of female rats: reproductive organ response variations with stage of estrus. Experimental Neurology 210 375-387.

Collins JJ, Lin CE, Berthoud HR \& Papka RE 1999 Vagal afferents from the uterus and cervix provide direct connections to the brainstem. Cell Tissue Research 295 43-54. 
Cueva-Rolon R, Sansone G, Bianca R, Gomez LE, Beyer C, Whipple B \& Komisaruk BR 1996 Vagotomy blocks responses to vaginocervical stimulation after genitospinal neurectomy in rats. Physiology \& Behavior 60 19-24.

Cummings BJ \& Cotman CW 1995 Image analysis of beta-amyloid load in Alzheimer's disease and relation to dementia severity. Lancet $\mathbf{3 4 6}$ $1524-1528$.

Enquist LW 2002 Exploiting circuit-specific spread of pseudorabies virus in the central nervous system: insights to pathogenesis and circuit tracers. Journal of Infectious Diseases 186 (Supplement 2) S209-S214.

Francis K, Meddle SL, Bishop VR \& Russell JA 2002 Progesterone receptor expression in the pregnant and parturient rat hypothalamus and brainstem. Brain Research 927 18-26.

Giesler GJ Jr, Nahin RL \& Madsen AM 1984 Postsynaptic dorsal column pathway of the rat. I. Anatomical studies. Journal of Neurophysiology $\mathbf{5 1}$ 260-275.

Haase EB, Buchman J, Tietz AE \& Schramm LP 1997 Pregnancy-induced uterine neuronal degeneration in the rat. Cell Tissue Research $\mathbf{2 8 8}$ 293-306.

Higuchi T \& Okere CO 2002 Role of the supraoptic nucleus in regulation of parturition and milk ejection revisited. Microscopy Research and Technique 56 113-121.

Higuchi T, Uchide K, Honda K \& Negoro H 1987 Pelvic neurectomy abolishes the fetus-expulsion reflex and induces dystocia in the rat. Experimental Neurology 96 443-455.

Hubscher CH \& Berkley KJ 1995 Spinal and vagal influences on the responses of rat solitary nucleus neurons to stimulation of uterus, cervix and vagina. Brain Research 702 251-254.

Jancso G 1992 Pathobiological reactions of C-fibre primary sensory neurones to peripheral nerve injury. Experimental Physiology 77 405-431.

Kirby LS, Kirby MA, Warren JW, Tran LT \& Yellon SM 2005 Increased innervation and ripening of the prepartum murine cervix. Journal of the Society for Gynecologic Investigation 12 578-585.

Kirby MA, Groves M \& Yellon SM 2009 Retrograde tracing of spinal cord connections to the cervix with pregnancy in mice. Reproduction 139 645-653.

Klein DC, Smoot R, Weller JL, Higa S, Markey SP, Creed GJ \& Jacobowitz DM 1983 Lesions of the paraventricular nucleus area of the hypothalamus disrupt the suprachiasmatic leads to spinal cord circuit in the melatonin rhythm generating system. Brain Research Bulletin 10 647-652.

Klukovits A, Gaspar R, Santha P, Jancso G \& Falkay G 2002 Functional and histochemical characterization of a uterine adrenergic denervation process in pregnant rat. Biology of Reproduction 67 1013-1017.

Kobayashi Y 1998 Distribution and morphology of spinothalamic tract neurons in the rat. Anatomica Embryologica 197 51-67.

Komisaruk BR \& Sansone G 2003 Neural pathways mediating vaginal function: the vagus nerves and spinal cord oxytocin. Scandinavian Journal of Psychology $\mathbf{4 4}$ 241-250.

Komisaruk BR, Bianca R, Sansone G, Gomez LE, Cueva-Rolon R, Beyer C \& Whipple B 1996 Brain-mediated responses to vaginocervical stimulation in spinal cord-transected rats: role of the vagus nerves. Brain Research 708 128-134.

Krukoff TL 1998 Central regulation of autonomic function: no brakes? Clinical and Experimental Pharmacology \& Physiology 25 474-478.

Lee JW \& Erskine MS 2000 Pseudorabies virus tracing of neural pathways between the uterine cervix and CNS: effects of survival time, estrogen treatment, rhizotomy, and pelvic nerve transection. Journal of Comparative Neurology 418 484-503.

Leppert PC 1995 Anatomy and physiology of cervical ripening. Clinical Obstetrics and Gynecology 38 267-279.

Longo LD \& Yellon S 1988 Biological timekeeping during pregnancy and the role of circadian rhythms in parturition. In The Endocrine Control of the Fetus, pp 173-191. Eds W Kunzel \& A Jensen. Berlin: Springer-Verlag.

Mackay LB, Shi SQ, Garfield RE \& Maner WL 2009 The effect of bilateral pelvic neurectomy on uterine and abdominal electrical and pressure activity, as measured by telemetry in conscious, unrestrained pregnant rats. Journal of Perinatal Medicine 37 313-319.

Mackler AM, lezza G, Akin MR, McMillan P \& Yellon SM 1999 Macrophage trafficking in the uterus and cervix precedes parturition in the mouse. Biology of Reproduction 61 879-883.
Numan M, Roach JK, del Cerro MC, Guillamon A, Segovia S, Sheehan TP \& Numan MJ 1999 Expression of intracellular progesterone receptors in rat brain during different reproductive states, and involvement in maternal behavior. Brain Research 830 358-371.

Nylen A, Skagerberg G, Alm P, Larsson B, Holmqvist B \& Andersson KE 2001 Nitric oxide synthase in the hypothalamic paraventricular nucleus of the female rat; organization of spinal projections and coexistence with oxytocin or vasopressin. Brain Research 908 10-24.

Ortega-Villalobos M, Garcia-Bazan M, Solano-Flores LP, NinomiyaAlarcon JG, Guevara-Guzman R \& Wayner MJ 1990 Vagus nerve afferent and efferent innervation of the rat uterus: an electrophysiological and HRP study. Brain Research Bulletin 25 365-371.

Papka RE, Williams S, Miller KE, Copelin T \& Puri P 1998 CNS location of uterine-related neurons revealed by trans-synaptic tracing with pseudorabies virus and their relation to estrogen receptor-immunoreactive neurons. Neuroscience 84 935-952.

Paxinos G \& Franklin KBJ 2004 The Mouse Brain, New York: Elsevier.

Puder BA \& Papka RE 2001 Hypothalamic paraventricular axons projecting to the female rat lumbosacral spinal cord contain oxytocin immunoreactivity. Journal of Neuroscience Research 64 53-60.

Reppert SM, Henshaw D, Schwartz WJ \& Weaver DR 1987 The circadiangated timing of birth in rats: disruption by maternal SCN lesions or by removal of the fetal brain. Brain Research 403 398-402.

Russell JA, Leng G \& Douglas AJ 2003 The magnocellular oxytocin system, the fount of maternity: adaptations in pregnancy. Frontiers in Neuroendocrinology 24 27-61.

Sawchenko PE \& Swanson LW 1983 The organization and biochemical specificity of afferent projections to the paraventricular and supraoptic nuclei. Progress in Brain Research 60 19-29.

Schwanzel-Fukuda M, Morrell JI \& Pfaff DW 1984 Localization of forebrain neurons which project directly to the medulla and spinal cord of the rat by retrograde tracing with wheat germ agglutinin. Journal of Comparative Neurology 226 1-20.

Sidman RL, Angevine JB Jr \& Pierce ET 1983 Atlas of the Mouse Brain and Spinal Cord. Cambridge: Harvard University Press.

Silverman AJ \& Pickard GE 1983 The hypothalamus. In Chemical Neuroanatomy, pp 295-336. Ed. PC Emson. New York: Raven Press.

Simerly RB 1995 Anatomical substrates of hypothalamic integration. In The Rat Nervous System, pp 353-376. Ed. G Paxinos. San Diego, CA: Academic Press.

Steinman JL, Carlton SM \& Willis WD 1992 The segmental distribution of afferent fibers from the vaginal cervix and hypogastric nerve in rats. Brain Research 575 25-31.

Strack AM \& Loewy AD 1990 Pseudorabies virus: a highly specific transneuronal cell body marker in the sympathetic nervous system. Journal of Neuroscience 10 2139-2147.

Swanson LW \& Cowan WM 1975 The efferent connections of the suprachiasmatic nucleus of the hypothalamus. Journal of Comparative Neurology 160 1-12.

Swanson LW \& Sawchenko PE 1983 Hypothalamic integration: organization of the paraventricular and supraoptic nuclei. Annual Review of Neuroscience 6 269-324.

Tingaker BK, Ekman-Ordeberg G \& Forsgren S 2006 Presence of sensory nerve corpuscles in the human corpus and cervix uteri during pregnancy and labor as revealed by immunohistochemistry. Reproductive Biology and Endocrinology 445.

Toth IE \& Palkovits M 1997 Viral labelling of synaptically connected neurons. Neurobiology 5 17-41.

Van den Pol AN 1982 The magnocellular and parvocellular paraventricular nucleus of rat: intrinsic organization. Journal of Comparative Neurology 206 317-345.

Virgo BB \& Bellward GD 1974 Serum progesterone levels in the pregnant and postpartum laboratory mouse. Endocrinology 95 1486-1490.

Wagner CK \& Clemens LG 1991 Projections of the paraventricular nucleus of the hypothalamus to the sexually dimorphic lumbosacral region of the spinal cord. Brain Research 539 254-262.

Warembourg M 1978 Uptake of (3)H labeled synthetic progestin by rat brain and pituitary. A radioautography study. Neuroscience Letters 9 329-332.

Weaver DR 1998 The suprachiasmatic nucleus: a 25-year retrospective. Journal of Biological Rhythms 13 100-112. 
Wiesel O, Toth IE, Boldogkoi Z, Hornyak A, Bokor V, Halasz B \& Gerendai I 2004 Comparison of transsynaptic viral labeling of central nervous system structures from the uterine horn in virgin, pregnant, and lactating rats. Microscopy Research and Technique $63244-252$.

Wikland M, Lindblom B, Dahlstrom A \& Haglid KG 1984 Structural and functional evidence for the denervation of human myometrium during pregnancy. Obstetrics and Gynecology 64 503-509.

Yellon SM \& Longo LD 1987 Melatonin rhythms in fetal and maternal circulation during pregnancy in sheep. American Journal of Physiology 252 E799-E802.

Yellon SM, Mackler AM \& Kirby MA 2003 The role of leukocyte traffic and activation in parturition. Journal of the Society for Gynecologic Investigation 10 323-338.

Yellon SM, Ebner CA \& Sugimoto Y 2008 Parturition and recruitment of macrophages in cervix of mice lacking the prostaglandin $\mathrm{F}$ receptor. Biology of Reproduction 78 438-444.
Yellon SM, Lechuga TJ, Burns AE \& Kirby MA 2009 Transection of the vagus nerve delays birth and alters cervical ripening in rat. Reproductive Sciences 16 67A-374A. Page 302A, abstract 808.

Zimmerman EA, Stillman MA, Recht LD, Antunes JL, Carmel PW \& Goldsmith PC 1977 Vasopressin and corticotropin-releasing factor: an axonal pathway to portal capillaries in the zona externa of the median eminence containing vasopressin and its interaction with adrenal corticoids. Annals of the New York Academy of Sciences 297 405-419.

Received 4 January 2010

First decision 12 February 2010

Revised manuscript received 26 March 2010

Accepted 6 May 2010 